\title{
Discussion on Standardization of Metering Interface for Metering Unit and Electronic Transformer
}

Mai Zhao Jiao

\section{Power Metering Center, Hainan Power Grid Co., Ltd., Haikou, Hainan 570100}

Abstract: Electronic transformers play a very important role in the practice, but because the manufacturers of electronic transformers are relatively diverse, so they produce the type of electronic transformers are endless, which makes the electronic There are some differences in the interface of the transformer. To protect the role of electronic transformers play, in order to enhance the practical application of electronic transformers, electronic output of the transformer should also be a scientific measurement, the electronic transformer interface unified model.

\section{Key words: metering with the unit; metering} interface standardization

\section{The measurement with the merging unit}

Electronic transformers play a very important role in the construction of power grids, but because the interface of electronic transformers is not standardized, it limits its function to play. Therefore, in the use of electronic transformers, the need for the use of metering unit with the method.

\subsection{The concept of metering with the unit}

In the grid system, the role of electronic transformers is very important, electronic transformers generally through the measurement channel to play a role ${ }^{1}$. However, the use of electronic transformers in the different aspects of the grid, its metering channel will appear inconsistent, the method used by the way there are significant differences. In different channels, the value of the merging unit is also a large difference, this value is mainly refers to the discrete value, rather than other values. In general, in the protection channel, its value is relatively small. But in the measurement channel, this value is relatively large. In this regard, the use of electronic transformers in the process, the need to combine different channels, the use of different measurement methods. Of course, in order to regulate the use of electronic transformers, you need to use the metering unit with the way.

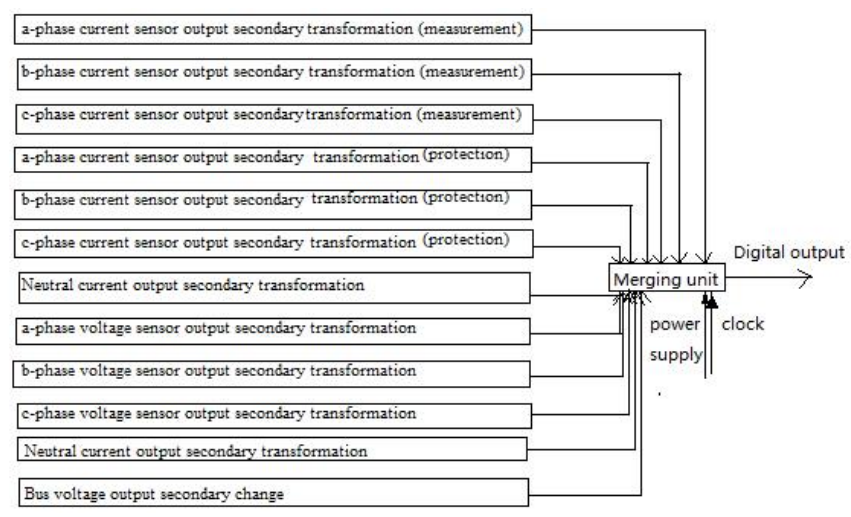

Figure: The way of metering merging unit function

\subsection{Management of metering units}

In the process of grid construction, the use of electronic transformers and metering units with the way to the scientific calculation of electricity, can accurately implement the scientific budget of electricity. In this regard, in the power grid system, the electronic transformer and metering unit can play a key role in the way, can enhance the scientific nature of energy calculation, which requires the use of metering unit with the way to achieve uniformity of the interface specification ${ }^{2}$. On the one hand, in the measurement of the use of merging unit management, should be used full-time management methods, arrangements for professionals to conduct unified management. This management can strengthen the efficiency of management, improve the quality of management, optimize the management level, but also to manage the duties of the implementation of the individual head, urging them to seriously perform their duties, to ensure that the role of measurement with the merger unit play. On the other hand, in the 
actual management, should also implement a more scientific, more refined encryption management. Non-system internal staff, no one has the right to unauthorized equipment, no person, etc. shall not be in private contact equipment. Through this management, can put an end to human reasons or man-made errors, resulting in the accuracy of its measurement accuracy of the serious consequences.

\section{The electronic transformer metering interface standardization analysis}

Electronic transformers play an important role in the grid system, but because of the different types of electronic transformer manufacturers, the type of electronic transformer produced by the different, which to some extent caused by the electronic transformer Interface inconsistencies, a direct result of the late measurement of the differences and mistakes. Therefore, it is necessary to strengthen the standardization of the electronic transformer interface, the use of reasonable measurement with the merger unit method to optimize the unity of measurement and normative ${ }^{3}$.

\subsection{Analog signal output interface specification}

In the use of electronic transformers, although it is on the surface and the merger unit is divided into different systems, but in fact the two are closely related. In the power grid, the use of electronic transformers need to simulate the output of the signal, the general use of the same specifications and models of the interface, this can guarantee the consistency of signal output, easy to measure and statistics later. In the signal simulation stage, should be a comprehensive analysis and screening to protect the signal output stability and reliability, to ensure the accuracy of signal output and scientific. The key is that the use of electronic transformers in the process, the output interface capacity is limited, coupled with the value of the merging unit, often increase the output interface load. Therefore, in the process of outputting the signal, the overall capacity of the output interface should be analyzed to ensure that the output value is within the range of the interface load. In addition, in the actual use of the process, the need to combine the actual situation, the classification of different electronic transformer use.

\subsection{The specification of digital output interface}

In the use of electronic transformers, especially in the stage of its signal transmission, it can often be divided into two ways, digital output and physical interface at the same time the use of the way. These two methods in the use of the process, respectively, have their own characteristics and advantages. The former in the actual use of the process, the transmission capacity is very large, and the transmission efficiency is particularly high, the entire transmission process is automated. Compared to the former, the latter is a more traditional way. In the analysis of the ability of digital output, should use the scientific norms, but also should focus on the overall accuracy of digital output and stability.

\section{The electronic transformer and the merging unit with the docking}

In the electronic transformer, the merging unit plays a key role, is the electronic transformer "central nervous system." Electronic transformer and the merging unit between the reasonable match and docking, can give full play to the role of electronic transformers. On the one hand, the realization of electronic transformers and merging unit with the need to deal with interface problems. The merging unit is a current/voltage transformer for digital output. When the merging unit plays a role, the 7-channel current signal can be combined into one unit group, and the data transmission can be realized. It can be seen that in the electronic transformer, the role of the merging unit is the standardization and standardization of the data interface. In the process of signal transmission, the signal processing capacity of the merging unit is more prominent, the input (output) side can be an analog signal, and it can be a digital signal. On the other hand, in the docking process of the electronic transformer and the merging unit, the capacity of the electronic transformer is an upper limit. The merging unit can determine the capacity of the electronic transformer and transmit it in accordance with the normalized capacity.

\section{Conclusion}

The use of electronic transformers in the grid system is very extensive, but because of the different types of electronic transformers, so the interface is also different. Therefore, in actual use, in order to enhance the accuracy and accuracy of transmission, we must use the metering unit in this the way.

\section{References}

[1] Hu Haoliang, Wu Wei Jiang et al, Metering with the Unit and the Electronic Transformer Metering Interface Standardization [J]. Power grid technology, 2014,05: 1414-1419.

[2] Haoliang, H., Weijiang, W. and Min, L., 2014. Discussion on standardization of merging unit for 
metering and electronic transformer metering interface. Power System Technology, 38(5), pp.1414-1419.
[3] Analysis on Standardization of Metering Interface for Metering Unit and Electronic Transformer [J]. Power System Technology,2014,38(05):1414-1419. [2017-09-22]. DOI 10.13335/j.1000-3673.pst.2014.05.04 